\title{
Nonlocal effects of prosodic boundaries
}

\author{
Katy CARLSON \\ Morehead State University, Morehead, Kentucky \\ AND \\ Charles Clifton, Jr. and Lyn Frazier \\ University of Massachusetts, Amherst, Massachusetts
}

\begin{abstract}
Placing a prosodic boundary before a phrase may influence its syntactic analysis. However, the boundary's effect depends on the presence, size, and position of other, earlier, prosodic boundaries. In three experiments, we extend previous results about the effect of the position of the early boundary. In sentences in which a final phrase may modify either a local verb or an earlier verb, a boundary immediately after the first verb leads to more first-verb attachments than when the earlier boundary appears in another position between the two verbs (Experiments 1 and 2). This effect cannot be attributed to weaker effects of more distant boundaries (Experiment 2), but is likely due to the first verb being more prominent when a boundary immediately follows it, since similar effects are observed when the verb is accented (Experiment 3). The results support the informative boundary hypothesis and show that the impact of earlier, nonlocal boundaries is not fully uniform.
\end{abstract}

There has been considerable interest in the question of how intonation - in particular, prosodic boundariesinfluences the processing of a sentence (e.g., Beach, 1991; Kjelgaard \& Speer, 1999; Kraljic \& Brennan, 2005; Lehiste, 1973; Nespor \& Vogel, 1986; Price, Ostendorf, Shattuck-Hufnagel, \& Fong, 1991; Pynte \& Prieur, 1996; Schafer, Speer, Warren, \& White, 2000). The vast majority of researchers have examined sentences in which the presence or location of a single prosodic boundary affects the division of a sentence into syntactic phrases or affects the attachment of one phrase to others.

In our previous work, we investigated the effect of multiple prosodic boundaries, which led to the informative boundary hypothesis (Carlson, Clifton, \& Frazier, 2001; Clifton, Carlson, \& Frazier, 2002; cf. Schafer, 1997, for a position similar in some respects to ours). This hypothesis is that listeners interpret a prosodic boundary before a constituent that could be attached to either of two earlier constituents with reference to the presence and size of any relevant earlier boundary. For example, in Example 1 after John visited can be attached to the phrase headed by learned, where it modifies learned as in the paraphrase in Example 1a, or it can be attached to telephoned, for the meaning in Example 1b.

(1) Susie learned that Bill telephoned after John visited.

a. Susie learned (after John visited) that Bill telephoned.

b. Susie learned something - namely, that Bill telephoned after John visited.
If there are no relevant earlier boundaries, a boundary before the ambiguously attached constituent discourages local (low) attachment (see, e.g., Price et al., 1991). That is, a prosodic boundary immediately preceding after John visited may favor attachment to the high attachment site learned rather than to the low attachment site telephoned. But, according to the informative boundary hypothesis, if a (relevant) earlier prosodic boundary is larger than the boundary after telephoned, the prosody may still favor local attachment of after John visited to telephoned, even though a prosodic boundary intervenes. The informative boundary hypothesis takes a relevant earlier boundary to be a boundary that intervenes between the possible low and high attachment sites.

To specify the size of a boundary, we assume a phonological system that distinguishes word boundaries, intermediate phrase (ip) boundaries, and intonational phrase $(\mathrm{IPh})$ boundaries. An IPh is the largest unit, and it contains one or more ips. An ip must contain one or more words and at least one accented constituent. These prosodic units (as well as prosodic words) are identified following the ToBI system, a prosodic annotation procedure inspired by Pierrehumbert (1980) and explained in Beckman and Elam (1997). The ends of both types of prosodic phrases (IPhs and ips) are associated with tonal changes, increased duration, and optional pausing, with IPhs involving more extreme changes. This theory gives us a three-way distinction between prosodic boundary sizes: A word boundary is smaller than an ip boundary, which is smaller than an IPh boundary. Syntactic boundaries also differ in size, and their size primarily depends on which syntactic phrases

K. Carlson, k.carlson@moreheadstate.edu 
can be contained inside others: The edge of an entire clause is a larger syntactic boundary than the edge of one of its constituents, such as a noun phrase or a verb phrase.

The detailed motivation for the informative boundary hypothesis and some discussion of possible mechanisms are laid out in our earlier research (Carlson et al., 2001; Clifton et al., 2002; cf. Frazier, Carlson, \& Clifton, 2006, for general discussion), but - briefly - we view the presence of a prosodic boundary as evidence that a listener must interpret with respect to the local context, including the presence, size, and location of certain other prosodic boundaries. Our favored justification for this context sensitivity is that a listener assumes that speakers generally make their prosodic phrasing decisions conform to the intended structure and meaning of a sentence, differing primarily only where phonetic considerations dictate. For example, if a speaker reaches a syntactic boundary that is smaller than a previous syntactic boundary, the speaker will not capriciously produce a prosodic boundary that is larger than the prosodic boundary at the end of the larger constituent.

The informative boundary hypothesis has successfully predicted processing effects in various syntactic structures, shown in Example 2, with the relevant early and late boundary positions that we tested marked as \#1 and \#2 (Carlson et al., 2001; Clifton et al., 2002):

(2) a. Susie learned \#1 that Bill telephoned \#2 after John visited.

b. Old men \#1 and women \#2 with very large houses

c. Johnny \#1 and Sharon's \#2 in-laws

d. The daughter \#1 of the Pharaoh's \#2 son

e. I met the daughter \#1 of the colonel \#2 who was on the balcony.

In these sentences, a prosodic boundary of a given phonological size at position \#2 produced different results depending on whether this boundary was equal to, smaller than, or larger than a prosodic boundary at position \#1.

We propose that these effects arise not because prosodic boundaries send special signals to the syntactic processor (e.g., END A SYNTACTIC CONSTITUENT HERE; see Marcus \& Hindle, 1990), but because they create prosodic phrases that affect the likelihood of attachment. We claim that the processor prefers to syntactically group nearby constituents and constituents that are prosodically grouped together, if possible. If a constituent is in a different prosodic phrase from the nearest attachment site, it is less likely to attach locally than a constituent that is in the same prosodic phrase as the potential attachment site. The constituent is especially likely to attach nonlocally if the boundary that marks the end of the prosodic phrase containing the attachment site is larger than any other prosodic boundaries in the relevant area. In Example 2c, for example, an ip boundary at position \#2 would usually inhibit attachment of in-laws to Sharon's and favor attachment to the higher phrase Johnny and Sharon's. If an IPh boundary is also present at position \#1, however,
Sharon's and in-laws are more closely grouped prosodically than either one is to Johnny- $\left[(\text { Johnny })_{\mathrm{ip}}\right]_{\mathrm{IPh}}[$ (and Sharon's) $)_{\text {ip }}$ (in-laws) $\left.)_{\text {ip }}\right]_{I P h}$ - and low attachment becomes more attractive again (making the in-laws relatives only of Sharon). However, an earlier prosodic boundary that does not intervene between the potential attachment sites does not modulate the effect of the later boundary. Clifton et al. (2002) showed this for sentences like Example 2a, in which a prosodic boundary after Susie (instead of after learned) did not reduce the effect of a boundary after telephoned.

The informative boundary hypothesis denies the simple and attractive suggestion that a prosodic boundary is a local cue, signaling a listener not to attach an upcoming phrase to the immediately preceding material (Marcus \& Hindle, 1990; Watson \& Gibson, 2005). Marcus and Hindle stated that "major intonation boundaries are introduced into the lexical stream fed to the parser and ... the parser knows nothing about them. . . . Upon encountering an utterly unknown 'lexical' item [the boundary], a deterministic parser simply terminates the constituent at hand ...” (p. 495). Watson and Gibson (2005) explicitly proposed the "Anti-Attachment Hypothesis (AAH): Listeners prefer not to attach an incoming word to a lexical head that is immediately followed by an intonational boundary" (p. 285). ${ }^{1}$ In the work we report here, we explore a limitation on the informative boundary hypothesis - namely, the effect of placing an earlier prosodic boundary at different positions between the possible attachment sites. Even though these different positions are still relevant, according to our hypothesis, it turns out that they have somewhat different effects, which we explore in the present research.

In this article, we study a structure with two relevant early boundary positions, illustrated in Example 3 .

(3) Jimmy comforted \#1 the girl \#2 that he had insulted $\# 3$ at the end of the party.

In this structure, the temporal adjunct phrase at the end of the party could attach low- to the verb insulted inside the relative clause - or high - to the first verb comforted. Syntactic structures for these options are shown in Figure 1 . On any theory that considers only a local prosodic boundary, such as Watson and Gibson's (2005) AAH, a boundary at position \#3 should discourage local attachment of the adjunct regardless of the prosodic boundaries that might occur elsewhere in the utterance. According to our informative boundary hypothesis, the impact of a prosodic boundary at position \#3 (preceding the adjunct phrase) should be reduced by an equal or larger prosodic boundary at either of the two preceding positions, $\# 1$ or \#2. The presence of either early boundary should thus reduce the frequency of high attachments.

We report three experiments designed to help us understand the effects of boundaries at these different positions. In Experiment 1, we compared the impact of two relevant early boundaries: one immediately following the high attachment site (the first verb) and one occurring two words later, after the noun phrase that is the direct object of the first 

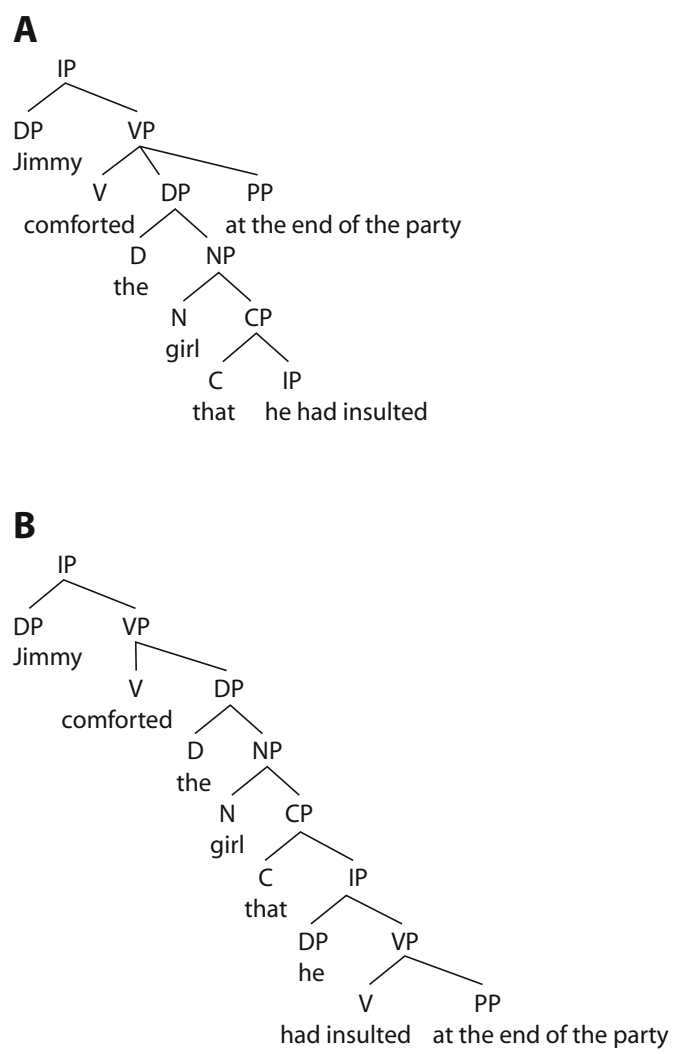

Figure 1. Syntactic structures for high (A) and low (B) attachment of the adjunct.

verb (Carlson et al., 2001, and Clifton et al., 2002, examined only early boundary positions directly after the high attachment site, with sentences that did not contain two relevant prosodically acceptable positions for boundaries). The results of Experiment 1 indicated that the impact of the two relevant early boundaries differed, a finding not predicted by the informative boundary hypothesis. Experiment 2 was designed to help us explore one account of this finding namely, that the distance between the early boundary and the later boundary affects the impact of the later boundary. In Experiment 3, we tested the hypothesis that the effect of the boundary immediately following the high attachment site was due to making that site prominent and salient.

To anticipate the results, we claim that our evidence indicates that a boundary immediately after an attachment site makes that site more salient and more attractive as an attachment site. This claim lets us explore the joint effects of prosodic boundaries and prominence on attachment decisions. Previous research has shown that the attachment of a constituent can be influenced not only by prosodic constituency, but also by prosodic prominence as manipulated by pitch accents (Beckman \& Elam, 1997). Schafer, Carter, Clifton, and Frazier (1996) studied the effects of pitch accents in the resolution of relative clause attachment, using phrases such as the propeller of the plane that was so shiny. The relative clause could attach to the nearest noun, plane, or to the more distant noun, propeller. Accenting either noun led to increased attachment of the relative clause to that noun, and the increase was larger if the accent was contrastive (i.e., a particularly high and steep accent, labeled as $\mathrm{L}+\mathrm{H}^{*}$ ).

Because prosodic boundaries often follow the most important accent in a phrase (Ladd, 1996; Selkirk, 1995), we hypothesize that the association between boundaries and accents might encourage a listener to interpret a prosodic boundary immediately after an attachment site as highlighting that site. That is, the boundary might give the impression of prominence even without a preceding accent. If this is true, Schafer et al.'s (1996) results suggest that when the first verb in our sentences (comforted in Example 3) is immediately followed by a prosodic boundary (position \#1), it will be perceived as having increased prominence and will attract attachment. Therefore, more high attachments will be observed when an early prosodic boundary follows the first verb (\#1) than when it occurs after a nonattachment site (\#2).

\section{EXPERIMENT 1}

In Experiment 1, we explored the effects of single and multiple prosodic boundaries on the attachment of temporal adjuncts in ambiguous sentences with restrictive relative clauses. We predicted that a single early prosodic boundary would result in numerous low attachments and that a single late boundary would result in frequent high attachments, whereas equal early and late boundaries would lead to intermediate results.

\section{Method}

Stimuli. We created 16 sentences, all ending in final temporal or locative phrases that could attach high or low. A full list of the stimuli is in Appendix A. These sentences were produced in four prosodic conditions, as in Example 4, with IPh boundaries at differing locations (indicated by //). IPh boundaries can involve different final tonal movements. The boundary tones that we used are those that are most consistent with sentence-internal phrases and form what is called a continuation rise, consisting of a low pitch target followed by a higher one (labeled as L-H\%).

(4) a. EarlyN: Jerry kissed the actress // that he had recently met at the Oscars ceremony.

b. Late: Jerry kissed the actress that he had recently met // at the Oscars ceremony.

c. EarlyV+ late: Jerry kissed // the actress that he had recently met // at the Oscars ceremony

d. EarlyN+late: Jerry kissed the actress // that he had recently met // at the Oscars ceremony.

The speaker, a native English speaker with training in ToBI analysis and experience recording experimental sentences, recorded the sentences and transferred them into a computer, digitized at 16 bits at a $22050-\mathrm{Hz}$ rate. They were examined in PRAAT (Boersma, 2001) to see that the intended prosodic contours had been achieved. Any anomalous sentences were rerecorded and analyzed. The pitch tracks for an example sentence can be seen in Figure 2, and average $f 0$ and duration measurements for critical sentence positions are in Tables 1 and $2 .{ }^{2}$ All of the sentences showed the $\mathrm{L}-\mathrm{H} \%$ contour diagnostic of an IPh boundary at the appropriate locations. 

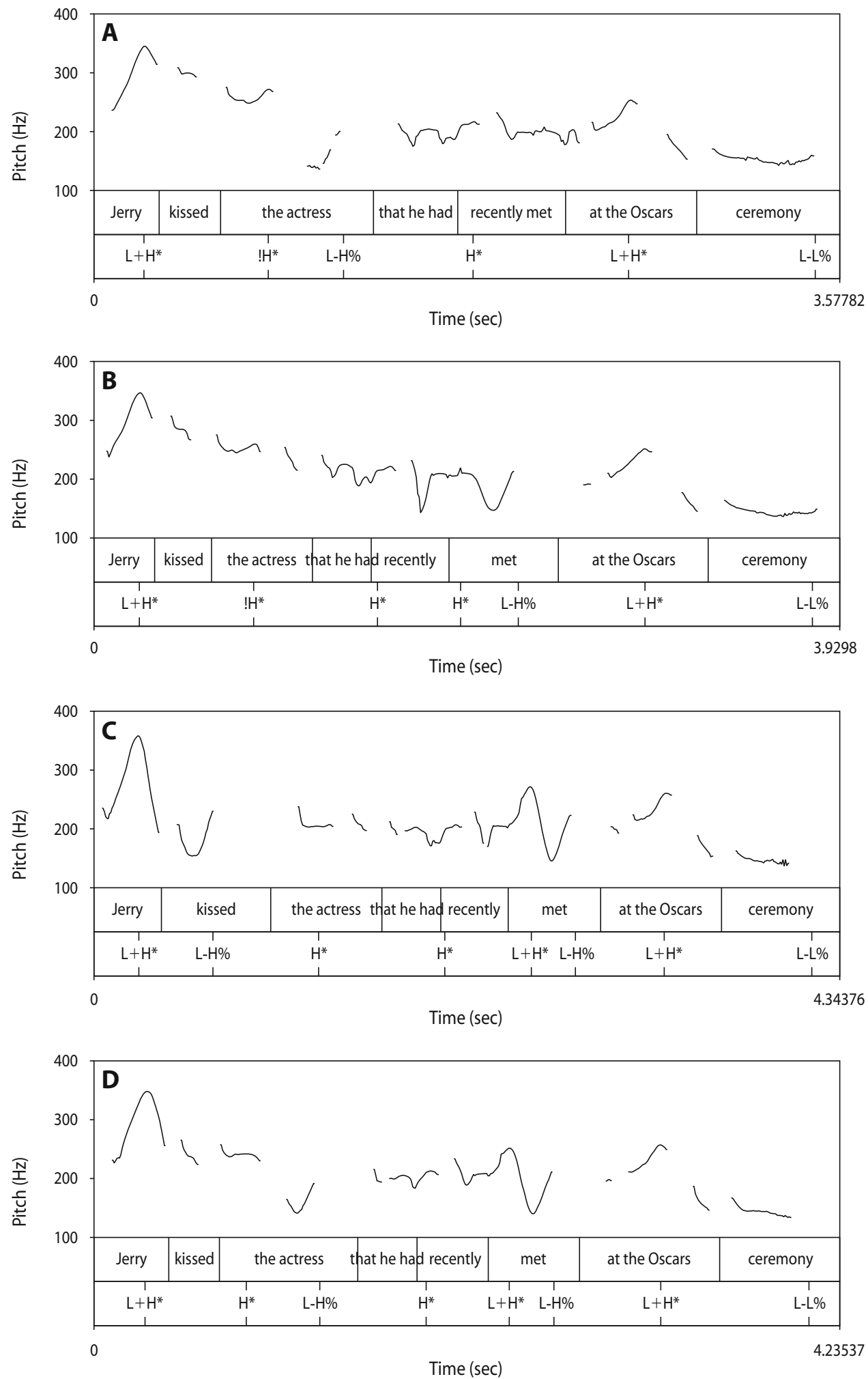

Figure 2. Pitch tracks for a sample item from Experiment 1. (A) EarlyN, (B) late, (C) earlyV+late, (D) earlyN+late. 
Table 1

Average Fundamental Frequency $(f 0)$ Measurements (in Hertz) for Boundary Positions, Experiments 1 and 2

\begin{tabular}{|c|c|c|c|c|c|c|}
\hline \multirow[b]{2}{*}{ Condition } & \multicolumn{2}{|c|}{ V1 } & \multicolumn{2}{|c|}{ N1 } & \multicolumn{2}{|c|}{$\mathrm{RC}$} \\
\hline & Peak & L-H\% & Peak & L-H $\%$ & Peak & L-H\% \\
\hline \multicolumn{7}{|c|}{ Experiment 1} \\
\hline EarlyN & 279 & $(252)$ & 240 & 141,191 & 214 & (198) \\
\hline Late & 276 & (259) & 243 & $(221)$ & 214 & 143,189 \\
\hline EarlyV+late & 231 & 147,200 & 218 & (195) & 244 & 143,194 \\
\hline EarlyN + late & 267 & $(253)$ & 238 & 141,188 & 242 & 140,185 \\
\hline \multicolumn{7}{|c|}{ Experiment 2} \\
\hline Short earlyV & 256 & 156,209 & 313 & $(253)$ & 247 & 151,207 \\
\hline Short earlyN & 294 & $(262)$ & 256 & 147,196 & 259 & 146,203 \\
\hline Long earlyV & 275 & 150,202 & 306 & $(243)$ & 233 & 146,203 \\
\hline Long earlyN & 300 & (269) & 258 & 144,196 & 246 & 145,199 \\
\hline
\end{tabular}

Note-Parenthetical numbers indicate $f 0$ averages over the few sentences that showed a discernible drop from the peak to the $f 0$ at the end of the word or constituent. In the other conditions, the two numbers indicate the successive low and high targets of the continuation rises $(\mathrm{L}-\mathrm{H} \%$, in ToBI transcription). V1, first verb; N1, noun modified by the relative clause; $\mathrm{RC}$, relative clause.

For each sentence, a follow-up question and two possible answers were created. The answers clarified the attachment of the adjunct phrase, as is shown in Example 5 with added parentheticals:

(5) What happened at the Oscars ceremony?
a. Jerry met the actress.
(low attachment)
b. Jerry kissed the actress.
(high attachment)

The same speaker who recorded the experimental sentences also recorded 104 other sentences that the participants were to hear. These sentences were a mixture of sentences with ambiguously interpreted ellipses and sentences with lexical ambiguities.

Participants. The participants were 28 native English-speaking students at Northwestern University who reported normal hearing and vision. They received course credit in an introductory linguistics class for participating in the experiment.

Procedure. The participants were seated wearing headphones at computer stations within a soundproof booth. They pressed a button on a keyboard to hear each sentence played by a computer. After the sentence ended, they read a corresponding question and answers on a written questionnaire sheet. They were to circle the answer that best fit how they understood the sentence and then press a button to hear the next item. A short practice round familiarized them with the procedure and allowed them to adjust the volume, if necessary. The entire procedure took less than half an hour.

The 16 experimental sentences (together with the 104 other sentences) were divided into four sets and assigned to four counterbalanced lists. Each counterbalanced list contained the four sentences of a set in one of the four prosodic conditions illustrated in Example 4, so that each list contained 4 sentences in each condition and each sentence appeared in all four forms across the lists. Seven participants heard each of the counterbalanced lists in a different pseudorandomized order with no consecutive trials containing sentences of the same type.

\section{Results}

The results are shown in Figure 3. As was expected, the condition with a single early prosodic boundary following the noun (earlyN) resulted in mostly low attachments. The condition with a single prosodic boundary immediately before the adjunct (late) resulted in mostly high attachments. The two conditions with double prosodic boundaries received intermediate results. These data were ana- lyzed as a mixed model logistic regression design, with both participants and items as random factors, treating the choice of interpretation as a binomial variable (see Jaeger, 2008, for justification). Since we are interested in all differences between individual conditions rather than the separate effects of the presence or absence of a boundary at the two locations, we treated the four conditions as a four-level fixed effect, permitting us to test the significance of each difference between a pair of conditions ordered in terms of increasing frequency of high attachments. Each condition differed from its nearest neighbors (and thus, from all other conditions). The earlyN+late condition resulted in more high attachments than the earlyN condition ( $49 \%$ and $26 \%$, respectively; $z=4.15$, $p<.001)$, the earlyV+late condition had more than the earlyN+late condition at $63 \%(z=6.23, p<.001)$, and the late condition $(79 \%)$ more than the earlyV + late condition $(z=8.38, p<.001)$.

\section{Discussion}

When a sentence contained a single prosodic boundary, its interpretation was largely determined by the position of the boundary. A late boundary immediately before the prepositional phrase (late) resulted in high attachments of the ambiguously attached prepositional phrase adjunct. An early boundary after the direct object noun (earlyN) resulted in low (local) attachments. Clearly, when the prosodic phrasing of the sentence grouped the adjunct with the immediately preceding material, the listeners preferred to attach the adjunct locally.

However, the apparent tendency for a boundary immediately before the adjunct to trigger high attachments was reduced when a second prosodic boundary separated the high attachment site and low attachment site. The frequency of high attachments was reduced by a second boundary in each of the two relevant positions, consistent with the informative boundary hypothesis. The reduction in frequency of high attachments was less, however, when the early boundary immediately followed the attachment site (earlyV+late) than when it came after the direct object noun (earlyN+late).

Table 2

Average Duration Measurements (in Milliseconds)

for Boundary Positions, Experiments 1 and 2

\begin{tabular}{|c|c|c|c|}
\hline & V1 & N1 & $\mathrm{V} 2$ \\
\hline \multicolumn{4}{|c|}{ Experiment 1} \\
\hline EarlyN & 388 & 763 & 483 \\
\hline Late & 396 & 397 & 933 \\
\hline EarlyV+late & 776 & 366 & 918 \\
\hline EarlyN + late & 360 & 760 & 920 \\
\hline \multicolumn{4}{|c|}{ Experiment 2} \\
\hline Short earlyV & 721 & 373 & 796 \\
\hline Short earlyN & 367 & 690 & 818 \\
\hline Long earlyV & 720 & 370 & 813 \\
\hline Long earlyN & 360 & 687 & 823 \\
\hline
\end{tabular}

Note-Duration measurements include the duration of the indicated words (V1, the first verb; N1, the noun modified by the relative clause; and V2, the second verb) plus any following pause. 


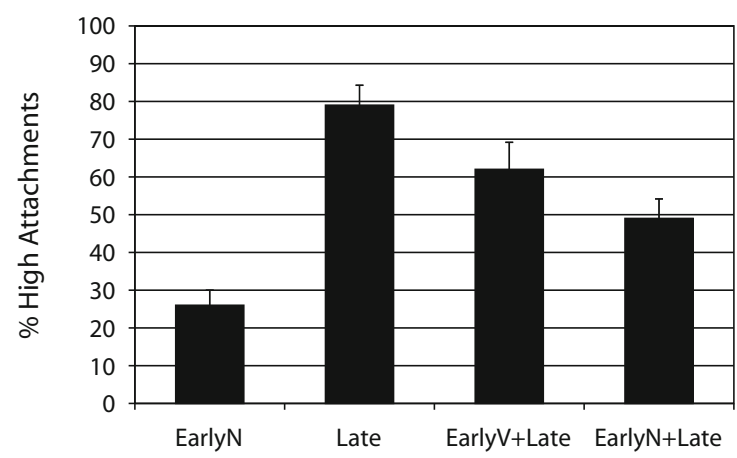

Figure 3. Percentage of high attachments, Experiment 1, with by-items standard error bars.

We will consider two possible reasons for this difference, which was not predicted by the informative boundary hypothesis. One possibility is that the greater distance between the early boundary and the critical late boundary in the early $\mathrm{V}+$ late condition resulted in decay of the memory representation of the size of the early boundary, thus lessening the impact on the interpretation of the late boundary. We tested this possibility in Experiment 2 by adding conditions in which the distance between the early and late boundaries is increased by adding a word or phrase inside the relative clause (after the earlyN boundary but before the late boundary). If increasing the distance between the boundaries lessens the impact of the early boundary on the late boundary, conditions with the word or phrase added should increase the frequency of high attachments.

A second possibility is that the greater frequency of high attachments for the early $\mathrm{V}+$ late condition than for the earlyN+late condition resulted from the postverbal boundary imparting prominence to the verb, making it a more attractive attachment site, as was discussed in the introduction. We tested this possibility in Experiment 3.

\section{EXPERIMENT 2}

In this experiment, we repeated the double-boundary conditions of Experiment 1 and added a phrase-length manipulation. We added a word or phrase inside the relative clause that modified the direct object (apparently in Example 6). The length of this word or phrase in syllables was comparable to how much farther away from the late boundary the early $\mathrm{V}$ boundary was, relative to the earlyN boundary. If the difference in the frequency of high attachments between the earlyV+late and early + late conditions in Experiment 1 was due solely to the extra distance between the boundaries in the former condition, a comparable difference in frequency of high attachments should result from the added length in the relative clause. Since boundary location and length were varied factorially in Experiment 2, one might expect their effects to combine in an additive or near-additive fashion.

\section{Method}

Stimuli. The 16 sentences from Experiment 1 were used as the short conditions in this experiment, and versions with lengthened relative clauses were compared with them. The length factor was crossed with the position of the early prosodic boundary, as in Example 6:

(6) a. Short earlyV+late: Brian interviewed// the diplomat that he had misquoted // before the last UN session.

b. Short earlyN+late: Brian interviewed the diplomat // that he had misquoted // before the last UN session.

c. Long earlyV+late: Brian interviewed // the diplomat that he had apparently misquoted // before the last UN session.

d. Long earlyN+late: Brian interviewed the diplomat // that he had apparently misquoted // before the last UN session.

The sentences all contained the late prosodic boundary immediately before the adjunct, so they were comparable to the doubleboundary conditions in the last experiment.

In Experiments 1 and 2, the early V boundary came an average of 3.25 syllables before the earlyN boundary. To ensure that the added length in the long relative clause condition resulted in a distance increase that was comparable to this, the material added to each relative clause was at least 3 syllables long and averaged 4.06 syllables (the short relative clauses averaged 5.88 syllables and the long 9.94 syllables). The short and long versions of the sentences are shown in Appendix B.

The four versions of the 16 sentences were rerecorded (by the same speaker as in Experiment 1), digitized at 16 bits and $22500 \mathrm{~Hz}$, and acoustically analyzed using PRAAT. As in the previous experiment, the prosodic boundaries in the sentences were continuation rise IPh boundaries. An example pitch track is shown in Figure 4, and average $f 0$ and duration measurements are in Tables 1 and 2 .

An additional 100 sentences (fillers and sentences from other experiments involving relative clause attachment ambiguities and ellipsis sentences with varying pitch accents) were recorded by the same speaker and digitized.

Participants. The participants in this experiment were 84 native English-speaking students with normal hearing at the University of Massachusetts, Amherst, fulfilling an experiment participation requirement for introductory psychology classes.

Procedure. The auditory questionnaire procedure was similar to that in the first experiment. Each participant sat in a soundproof booth and heard sentences played by a computer at a comfortable listening level over speakers. After a sentence was heard, the participant pulled a lever to indicate that he or she understood it, and then a written two-choice question (like those in Experiment 1) appeared on the computer screen. The participants answered the questions using response levers.

Four counterbalanced lists were created, as in Experiment 1. Each list contained 4 items in each of the four experimental conditions (plus the 100 other items), and each sentence was tested in each condition in one list. Thus, each participant heard four sentences in each of the four conditions. The items were presented in an individually randomized order, following a 5-item practice list. The entire procedure took less than half an hour.

\section{Results}

The results for Experiment 2 are shown in Figure 5. The data were analyzed as a mixed model logistic regression design, with both participants and items as random factors, treating the choice of interpretation as a binomial variable. The difference between the postverbal and postnominal early boundary position conditions was significant $(z=$ $3.18, p<.002$ ), with the postverbal early boundary lead- 

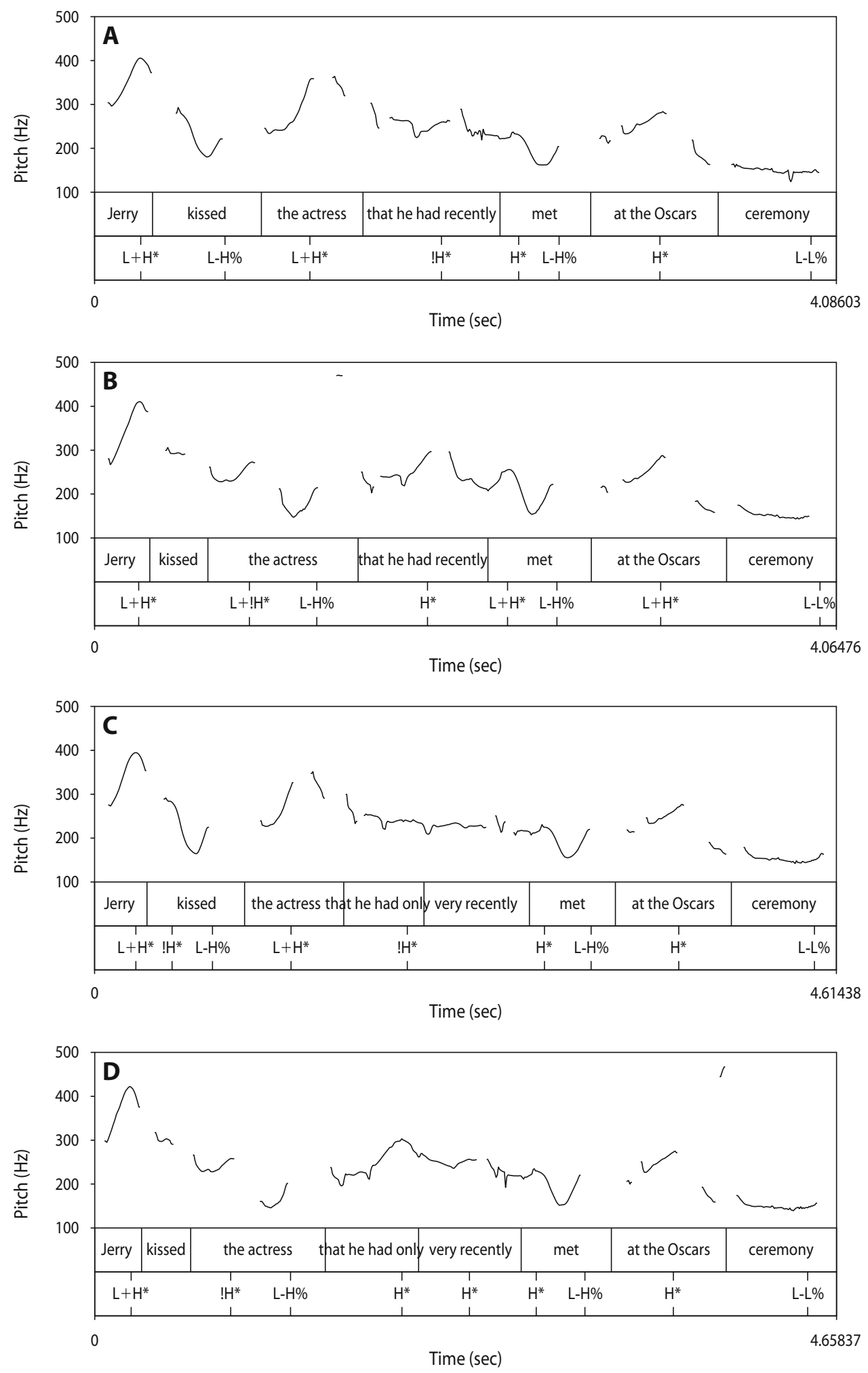

Figure 4. Pitch tracks for a sample item from Experiment 2. (A) Short earlyV, (B) short earlyN, (C) long earlyV, (D) long earlyN. 


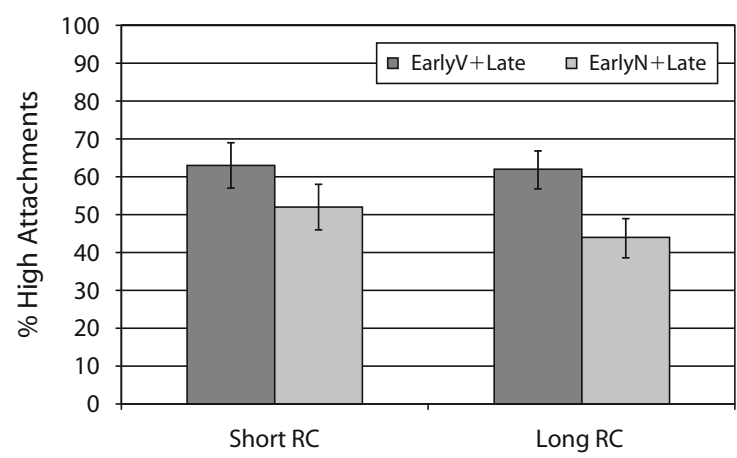

Figure 5. Percentage of high attachments, Experiment 2, with by-items standard error bars. RC, relative clause.

ing to more high attachments. The length manipulation resulted in a nonsignificant effect $(z<1.0)$, as did the interaction between length and boundary position $(z=$ $1.08, p>.27$ ).

Since the interaction between and the effects of short and long relative clauses were nonsignificant, any examination of the numerical difference between the two earlyN+late conditions must be considered suspect. With that warning in mind, we note that the apparent difference in frequency of high attachment interpretations between the long and short earlyN conditions (52\% vs. $44 \%$ ) was significant when tested both using a logistic mixed model $(z=2.1, p=.036)$ and using an ordinary $t$ test on participant means $[t(83)=2.1, p=.035]$. This difference is in the direction opposite to that predicted by the suggestion that increased distance between the boundaries results in a lessened impact of the early boundary on the late boundary. It cannot be taken as evidence that the effect of where the early boundary was placed in Experiment 1 is due to the distance between the early and late boundaries. If the difference is real, it may reflect some other effect of the length of the relative clause.

\section{Discussion}

As in Experiment 1, there were more high attachments when the early prosodic boundary followed the first verb (earlyV) than when it followed the noun (earlyN). The difference was again statistically significant, and in fact, the interpretation percentages for these double-boundary conditions were very similar in the two experiments. Length had no overall significant effect (and had a significant effect in the wrong direction in a follow-up contrast). This result argues against the possibility that the Experiment 1 observations that boundary \#1 had a smaller influence on the effect of the critical boundary \#3 than boundary $\# 2$ did were due to the greater distance of boundary \#1 from boundary \#3.

Before turning to an alternative account of the effect of early boundary position, we mention another possible basis for an effect of the length manipulation. It is possible that the sheer length of a phrase affects the informativeness of a prosodic boundary. One of the predictors of a prosodic boundary in production is a phonetically long upcoming or preceding constituent (Watson \& Gibson,
2004). Speakers are likely to place a prosodic boundary before or after a long constituent. In comprehension, Clifton, Carlson, \& Frazier (2006) showed that the effects of prosodic boundaries in coordination structures and complement structures could be weakened by increased constituent length: Listeners apparently interpreted the boundaries as reflecting phonetic, not syntactic, factors. If the $52 \%-44 \%$ difference between the long and short earlyN+late conditions in Experiment 2 is real, it could reflect such a factor. In the earlyN+late condition, the relative clause (that he had [apparently] misquoted in Example 6) without the ambiguously attached adjunct (before the last UN session) is bracketed by IPh boundaries. Perhaps making the relative clause longer by some four syllables justified these boundaries, reducing the tendency for the late boundary to encourage high attachment of the adjunct. We acknowledge that this suggestion is highly speculative, is based on shaky statistical evidence, and should only be taken to make the point that the interpretation of prosody is guided by multiple factors.

\section{EXPERIMENT 3}

In Experiment 3, we examined the possibility that the difference between the early + late and the early $\mathrm{V}+$ late conditions was evident because the boundary immediately after the verb in the latter condition made the verb more salient and available for attachments. As was noted earlier, prominent pitch accents are often followed by prosodic boundaries. A boundary might therefore highlight a preceding word even without a pitch accent present. If these speculations are on the right track, accenting this early verb might have the same effect on interpretations as another means of drawing attention to the actual high attachment site.

In this experiment, we followed up on the postverbal early boundary effect, testing whether accenting the first verb would increase high attachments to that verb. If so, this would support the suggestion that the earlyV+late condition in earlier experiments received more high attachments than the earlyN+late condition because the boundary after the verb made it more salient for attachment.

\section{Method}

Stimuli. The 16 sentences used in Experiment 1 (with a few words changed to increase comprehensibility; see Appendix A) were rerecorded with $\mathrm{H}^{*}$ accents on both verbs (comforted and insulted in Example 7), and with an ip boundary (indicated by /) before the final adverbial phrase (at the end of the party). A different speaker (also trained in prosodic phonology and the ToBI system) spoke the sentences. Each sentence was manipulated to create two different forms, one in which the $\mathrm{H}^{*}$ accent was eliminated from the first verb (comforted; Example 7a) and one in which the $\mathrm{H}^{*}$ accent was eliminated from the embedded relative clause verb (insulted; Example 7b).

(7) a. Embedded verb accent: Jimmy comforted the girl that he had insulted / at the end of the party.

b. First verb accent: Jimmy comforted the girl that he had insulted / at the end of the party.

The manipulation was performed in PRAAT (Boersma, 2001) using its manipulation, stylize pitch, and dur functions. The funda- 

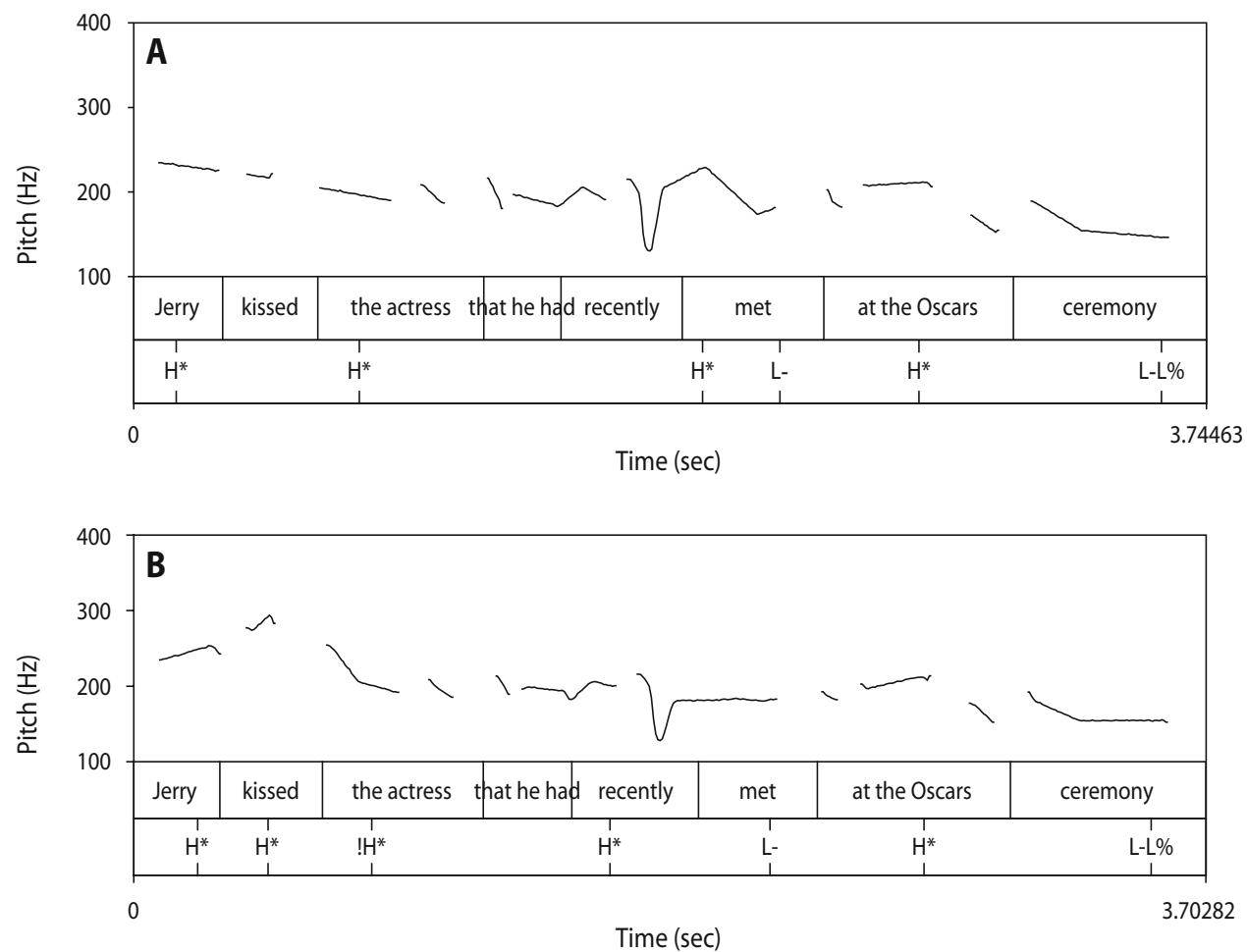

Figure 6. Pitch tracks for a sample item from Experiment 3. (A) Embedded verb accent, (B) first verb accent.

mental frequency contour of the sentence was linearly interpolated between inflection points (using a 1-semitone threshold for inflection points) and any pitch contour on the word whose $\mathrm{H}^{*}$ accent was to be eliminated was flattened by linear interpolation of pitch between the start and the end of the $\mathrm{H}^{*}$ pitch contour that peaked on the word (and frequently extended beyond it). The duration of the word was then shortened by increasing the rate of the word to $70 \%$ of its original duration at the midpoint of the word, with linear interpolation of rate adjustment to $100 \%$ at the start and end of the word. The sentence was then resynthesized using PRAAT's default PSOLA method. Sample pitch tracks for the two versions of 1 sentence appear in Figure 6, and the mean pitch and duration measures appear in Table 3 . The resulting sentences sounded reasonably natural, but to eliminate any possibility of the acoustic manipulations making them stand out in the experiment, one originally accented word from each filler and practice sentence was flattened in the same manner. ${ }^{3} \mathrm{An}$ additional 48 sentences were recorded ( 28 fillers and 20 sentences from an experiment on ambiguous ellipsis).

Participants. The participants in this experiment were 48 native English-speaking students at the University of Massachusetts, Amherst, with normal vision and hearing fulfilling an experiment participation requirement for introductory psychology classes.
Procedure. The procedure was the same as that in Experiment 2. Two counterbalanced lists were constructed, each with half of the experimental sentences in each condition. Half of the participants heard one list, and half the other. Each sentence was thus heard in each form by 24 participants. Each participant heard all of the items in the list in an individually randomized order, following a five-item practice list. The experiment took under $15 \mathrm{~min}$ and was followed by another, similarly short, experiment.

\section{Results}

The sentence-final adverbial was interpreted as modifying the first verb $69 \%$ of the time when the first verb retained its $\mathrm{H}^{*}$ accent and the relative clause verb was flattened and $62 \%$ of the time when the relative clause verb retained its $\mathrm{H}^{*}$ accent and the first verb was flattened. This difference, although small, was fully significant when tested using a logistic mixed model analysis (Jaeger, 2008; $z=2.16, p=.03$ ) and closely approached significance by a conventional test of the arcsin transformed proportions $\left[t_{1}(47)=1.93, p=.06\right.$, and $\left.t_{2}(15)=2.88, p=.011\right]$.

Table 3

Average $f 0$ Values (in Hertz) and Durations (in Milliseconds) for Selected Regions, Experiment 3

\begin{tabular}{lccccccc}
\hline & \multicolumn{3}{c}{ First Verb } & & \multicolumn{3}{c}{ Embedded Verb } \\
\cline { 2 - 4 } \cline { 6 - 8 } Verb Accent & Peak $f 0$ & End $f 0$ & Duration & & Peak $f 0$ & End $f 0$ & Duration \\
\hline Embedded & 227 & 210 & 380 & & 227 & 177 & 678 \\
First & 279 & 231 & 417 & & 195 & 173 & 610 \\
\hline
\end{tabular}




\section{Discussion}

The results of Experiment 3 indicate that an accented verb attracts an ambiguously attached adverbial modifier. In doing so, it supports the suggestion that placing a prosodic boundary immediately after the first verb increased its salience relative to placing the boundary after a following NP. It thus provides support for the possibility that the difference between the early $\mathrm{V}$ and earlyN conditions in Experiments 1 and 2 can be attributed to differences in the salience of a possible attachment site.

\section{GENERAL DISCUSSION}

In these experiments, we explored prosodic effects on the attachment of a temporal adjunct phrase to the verb of a preceding relative clause versus attachment to the main predicate. In the simplest cases, a prosodic boundary before the adjunct discouraged its attachment to the most local attachment site, the relative clause, whereas a single prosodic boundary early in the sentence encouraged that attachment. These results are consistent with theories about the effect of a single prosodic boundary on attachment (Watson \& Gibson, 2005). When prosodic boundaries of equal size were present at either of two positions before the adjunct and between the two attachment sites, however, the late boundary became less informative about attachment, leading to intermediate attachment percentages. This provides additional support for the informative boundary hypothesis (Carlson et al., 2001; Clifton et al., 2002).

In Experiment 1, a difference emerged between the effects of early boundaries at two different positions, such that an early prosodic boundary after the first verb led to more high attachment than did the other early boundary position. This result was replicated in Experiment 2. This finding was not predicted by the informative boundary hypothesis, and although it does not disconfirm the hypothesis, it indicates at least that other factors are also at work in guiding how prosody affects sentence comprehension.

Experiment 2 provided evidence that sheer distance between two prosodic boundaries is not the basis of the effect of the early boundary's position in Experiment 1 . Increasing the distance between the boundaries did not affect the impact of the early boundary on the late boundary in the way predicted by the suggestion that greater distance between boundaries lessens the impact of one on the other. This is an interesting result in itself, given that the distance between elements so often does affect sentence processing (e.g., Gibson, 1998).

Experiment 3 gave some support to the hypothesis that the boundary immediately after the high attachment site rendered the high attachment site (the first verb) more prominent or salient. It showed that accenting the first verb increased high attachments by a small amount. This finding is similar to Schafer et al.'s (1996) finding that an accent on one of two nouns attracts relative clause attachment.

The actual mechanisms by which pitch accents, and presumably prosodic boundaries, impart salience to a potential attachment site, and how salience affects inter- pretation, are uncertain. It is possible that the greater salience imparted by either manipulation simply makes the first verb more memorable and that participants are more likely to select an interpretation with a word that matches a word that is more available in memory. We do not consider this account very likely, since the participants were given two paraphrases to choose between, which placed little demand on memory for individual words. However (as pointed out by an anonymous reviewer), online studies that more directly assess how sentences are interpreted (as opposed to how responses are chosen) may be needed to dismiss the possibility definitively.

Assuming (as we do) that our participants actually understood the sentences and answered questions so as to reflect their understanding, various mechanisms remain possible. It might be that the presence of a prosodic boundary after a word imparts linguistic focus on that word, just as we assume a pitch accent does (Rooth, 1992). If this is so, a common mechanism could be involved in how prosodic boundaries and pitch accents affect sentence interpretation: They both affect focus, and focused words are good attachment sites. However, the effect of pitch accent was only about half the size of the effect of the prosodic boundary (measured as the difference in high attachment frequency between the early $\mathrm{V}+$ late and the early $\mathrm{N}+$ late conditions). This suggests that placing a boundary immediately after an attachment site may have some effect in addition to, or instead of, imparting focus.

A second question of mechanism involves how prosodic boundaries affect parsing. As was discussed earlier, some researchers have proposed that a prosodic boundary is used directly by the parser. The presence of a prosodic boundary signals a syntactic boundary, and stronger prosodic boundaries provide stronger signals. We advance a different position. We suggest that the syntactic parsing mechanism has access to how an utterance is divided into prosodic phrases. A prosodic boundary that is strong (in absolute terms or relative to other relevant boundaries) is more likely to signal the edge of a larger prosodic phrase, and the parser can map prosodic phrases onto syntactic phrases. Said simply, we propose that global prosodic structure, not local prosodic cues, affects sentence interpretation.

A final, very general, point: Prosodic structure does not map into syntactic structure in a simple, transparent fashion. Although some prosodic structures are incompatible with some syntactic structures, any given syntactic structure can generally be realized in different ways prosodically, and a given prosodic structure can be appropriate for various syntactic structures (see Frazier et al., 2006, for a discussion and references). Similarly, different aspects of prosody can convey different, and sometimes conflicting, information. Placing a boundary before a phrase, we claim, makes it likely that that phrase will be in a different prosodic constituent from the preceding word, decreasing the likelihood of attachment. However, a boundary can also increase the salience of the word that it immediately follows, increasing the likelihood of attachment to that word. Our evidence to date indicates that the former effect generally dominates, but additional study is clearly 
needed to determine how these distinct influences of prosodic boundaries are resolved.

\section{CONCLUSIONS}

The research reported here suggests that the overall prosodic contour of a sentence, including how boundaries are distributed and where pitch accents occur, can affect its interpretation (see also Schafer et al., 2000; Speer, Kjelgaard, \& Dobroth, 1996). This is in contrast to any view on which a prosodic event (boundary, accent) is viewed as affecting the syntactic structuring of a sentence in isolation in a context-independent and strictly local fashion (Marcus \& Hindle, 1990; Watson \& Gibson, 2005). We find that prosodic boundaries interact with each other and that a prosodic boundary can affect the salience of a preboundary attachment site.

\section{AUTHOR NOTE}

This research was supported in part by Kentucky NSF Grant EPSCoR REG EPS-0132295, University of Massachusetts Grant NSF-BCS 0090674, and Grant HD-18708 from the National Institute of Child Health and Human Development. The content is solely the authors' and does not reflect the official views of the National Institute of Child Health and Human Development or the National Institutes of Health. Correspondence concerning this article should be addressed to K. Carlson, Morehead State University, 150 University Blvd., Morehead, KY 40351 (e-mail: k.carlson@moreheadstate.edu).

\section{REFERENCES}

BEACH, C. M. (1991). The interpretation of prosodic patterns at points of syntactic structure ambiguity: Evidence for cue trading relations. Journal of Memory \& Language, 30, 644-663. doi:10.1016/0749 $-596 \mathrm{X}(91) 90030-\mathrm{N}$

Beckman, M. E., \& Elam, G. A. (1997). Guidelines for ToBI labelling, version 3.0. Retrieved April 20, 2009 from www.ling.ohio-state .edu/ tobi/ame_tobi/labelling_guide_v3.pdf.

BoERSMA, P. (2001). PRAAT, a system for doing phonetics by computer. Glot International, 5, 341-345.

Carlson, K., Clifton, C., JR., \& Frazier, L. (2001). Prosodic boundaries in adjunct attachment. Journal of Memory \& Language, 45, 5881. doi:10.1006/jmla.2000.2762

Clifton, C., Jr., Carlson, K., \& Frazier, L. (2002). Informative prosodic boundaries. Language \& Speech, 45, 87-114.

Clifton, C., JR., Carlson, K., \& Frazier, L. (2006). Tracking the what and why of speakers' choices: Prosodic boundaries and the length of constituents. Psychonomic Bulletin \& Review, 13, 854-861.

Frazier, L., Carlson, K., \& Clifton, C., Jr. (2006). Prosodic phrasing is central to language comprehension. Trends in Cognitive Sciences, 10, 244-249. doi:10.1016/j.tics.2006.04.002

Gibson, E. (1998). Linguistic complexity: Locality of syntactic dependencies. Cognition, 68, 1-76. doi:10.1016/S0010-0277(98)00034-1

JAEGER, T. F. (2008). Categorical data analysis: Away from ANOVAs (transformation or not) and towards logit mixed models. Journal of Memory \& Language, 59, 434-446. doi:10.1016/j.jml.2007.11.007

KJelgaard, M. M., \& Speer, S. R. (1999). Prosodic facilitation and interference in the resolution of temporary syntactic closure ambiguity. Journal of Memory \& Language, 40, 153-194. doi:10.1006/ jmla.1998.2620

Kralitc, T., \& Brennan, S. E. (2005). Prosodic disambiguation of syntactic structure: For the speaker or for the addressee? Cognitive Psychology, 50, 194-231. doi:10.1016/j.cogpsych.2004.08.002

LADD, D. R. (1996). Intonational phonology. Cambridge: Cambridge University Press.
LEE, E.-K., \& WATSON, D. G. (2009, March). Do listeners keep track of global prosodic information? Evidence from an eye-tracking study. Poster presented at CUNY Conference on Human Sentence Processing, Davis, CA

Lehiste, I. (1973). Phonetic disambiguation of syntactic ambiguity. Glossa, 7, 102-122.

Marcus, M., \& Hindle, D. (1990). Description theory and intonation boundaries. In G. Altmann (Ed.), Cognitive models of speech processing: Psycholinguistic and computational perspectives ( $\mathrm{pp} .483-512$ ). Cambridge, MA: MIT Press.

Nespor, M. A., \& Vogel, I. (1986). Prosodic phonology. Boston: Kluwer.

Pierrehumbert, J. (1980). The phonology and phonetics of English intonation. Unpublished doctoral dissertation, Massachusetts Institute of Technology, Cambridge.

Price, P. J., Ostendorf, M., Shattuck-Hufnagel, S., \& Fong, C. (1991). The use of prosody in syntactic disambiguation. Journal of the Acoustical Society of America, 90, 2956-2970. doi:10.1121/1.401770

Pynte, J., \& Prieur, B. (1996). Prosodic breaks and attachment decisions in sentence parsing. Language \& Cognitive Processes, 11, 165192. doi:10.1080/016909696387259

Rоoтн, M. (1992). A theory of focus interpretation. Natural Language Semantics, 1, 75-116.

SCHAFER, A. J. (1997). Prosodic parsing: The role of prosody in sentence comprehension. Unpublished doctoral dissertation, University of Massachusetts, Amherst.

Schafer, A. J., Carter, J., Clifton, C., JR., \& Frazier, L. (1996). Focus in relative clause construal. Language \& Cognitive Processes, 11, 135-163. doi:10.1080/016909696387240

Schafer, A. J., Speer, S. R., Warren, P., \& White, S. D. (2000). Intonational disambiguation in sentence production and comprehension. Journal of Psycholinguistic Research, 29, 169-182. doi:10.1023/A:1005192911512

SELKIRK, E. O. (1995). Sentence prosody: Intonation, stress, and phrasing. In J. Goldsmith (Ed.), The handbook of phonological theory (pp. 550-569). Oxford: Blackwell.

Speer, S. R., KJelgaArd, M. M., \& Dobroth, K. M. (1996). The influence of prosodic structure on the resolution of temporary syntactic closure ambiguities. Journal of Psycholinguistic Research, 25, 249271. doi:10.1007/BF01708573

WATSON, D., \& GiBSON, E. (2004). The relationship between intonational phrasing and syntactic structure in language production. Language \& Cognitive Processes, 19, 713-755. doi:10.1080/01690960444000070

WATsON, D., \& GiBson, E. (2005). Intonational phrasing and constituency in language production and comprehension. Studia Linguistica, $\mathbf{5 9}, 279-300$.

\section{NOTES}

1. Lee and Watson (2009) recently presented evidence that, in directive sentences like Click on the candle below the triangle that's in the blue circle, the effect of a prosodic boundary before that's on the blue circle is not affected by the size of a prosodic boundary after candle. They argued that this finding contradicts the informative boundary hypothesis. We are not yet able to explain why Lee and Watson's results differed from the results that we obtained with a similar relative clause attachment ambiguity (Example 2e), and we view the Lee and Watson finding as a not-yet-understood limitation on the hypothesis.

2. Although a prosodic boundary after the head noun of a relative clause (as in Examples 4a and 4d) is required for nonrestrictive relative clauses, it is also possible for restrictive relative clauses. The relative pronoun that, found in most of the relative clauses in these sentences, unambiguously indicates that they must be restrictive. It's also unclear how attachment of a later constituent would be affected by the status of the relative clause in any case.

3 . Because of experimenter error, the embedded verb was not deaccented in the first verb accent condition for one sentence. However, this error should only have reduced the size of the effect of accenting the first versus the embedded verb, and the sentence in question produced an interpretive difference that was typical of the remaining sentences. 


\section{APPENDIX A \\ Sentences for Experiments 1 and 3 (Alternative Phrases \\ Used in Experiment 3 Indicated After the Slashes)}

1. Jimmy comforted the girl that he \{got engaged to/danced with $\}$ at the end of the party.

2. Sarah watched the lawyer that she had danced with for several hours last night.

3. \{Elias/Alan $\}$ avoided the banker that he borrowed from long before the deadline.

4. $\{$ Jared/Jason $\}$ hired the secretary he had been mean to after the business folded.

5. Diane charmed the landlord that she \{reprimanded/badmouthed $\}$ at the tenants' meeting.

6. Karen called the chef that she had complimented after the year-end banquet.

7. Brian interviewed the diplomat that he had misquoted before the last UN session.

8. Nancy \{sketched/called\} the model that she almost hired for a crowd scene in the painting.

9. Jerry kissed the actress that he had recently met at the Oscars ceremony.

10. Brenda snubbed the waiter that $\{$ she took a dislike to/she'd been rude to $\}$ at the post-elopement dinner.

11. Gary robbed the professor that he $\{$ washed windows/worked $\}$ for during spring vacation.

12. Hazel placated the critic that she had publicly slandered before the summer season.

13. Chester \{rewarded/thanked\} the janitor that he left such a mess for after the carpet installation.

14. Peggy applauded the juggler that she could see best after the flaming baton act.

15. Earl pitied the students that he sent outside after the sudden downpour.

16. Amber recognized the picketer that she photographed during the political rally.

\section{Sentences for Experiment 2: Short Then Long Versions}

1. Jimmy comforted the girl that he insulted at the end of the party. Jimmy comforted the girl that he so thoughtlessly insulted at the end of the party.

2. Sarah watched the lawyer that she had danced with for several hours last night. Sarah watched the lawyer that she had so energetically danced with for several hours last night.

3. Elias avoided the banker that he borrowed from long before the deadline. Elias avoided the banker that he borrowed lots of money from long before the deadline.

4. Jared hired the secretary that he had been mean to after the business folded. Jared hired the secretary that he had been so nasty and mean to after the business folded.

5. Diane charmed the landlord that she reprimanded at the tenants' meeting. Diane charmed the landlord that she had indiscreetly reprimanded at the tenants' meeting.

6. Karen called the chef that she had complimented after the year-end banquet. Karen called the chef that she had so profusely complimented after the year-end banquet.

7. Brian interviewed the diplomat that he had misquoted before the last UN session. Brian interviewed the diplomat that he had apparently misquoted before the last UN session.

8. Nancy called the model that she almost hired for a magazine layout at breakfast time. Nancy called the model that she interviewed and almost hired for a magazine layout at breakfast time.

9. Jerry kissed the actress that he had recently met at the Oscars ceremony. Jerry kissed the actress that he had only very recently met at the Oscars ceremony.

10. Brenda snubbed the waiter that she took a dislike to at the pre-wedding dinner. Brenda snubbed the waiter that she immediately took a dislike to at the pre-wedding dinner.

11. Gary robbed the professor that he worked for during spring vacation. Gary robbed the professor that he and his roommates worked for during spring vacation.

12. Hazel placated the critic that she had publicly slandered before the summer season. Hazel placated the critic that she had shamelessly and publicly slandered before the summer season.

13. Chester rewarded the janitor that he left such a mess for after the carpet installation. Chester rewarded the janitor that he left such a large and disgusting mess for after the carpet installation.

14. Peggy applauded the juggler that she could see best after the flaming baton act. Peggy applauded the juggler that she and her friends could see best after the flaming baton act.

15. Earl pitied the students that he sent outside after the sudden downpour. Earl pitied the students that he thoughtlessly sent outside after the sudden downpour.

16. Amber recognized the picketer that she photographed during the political rally. Amber recognized the picketer that she carefully photographed during the political rally. 